\title{
Residual Current Devices
}

\begin{tabular}{|l|r|}
\hline Surnames, name & Antonino Daviu, Jose Alfonso (joanda@die.upv.es) \\
\hline Department & Departamento de Ingeniería Eléctrica \\
\hline Centre & Universitat Politècnica de València \\
\hline
\end{tabular}




\section{Summary}

The aim of this paper is to present a basic introduction to the operation of the residual current devices (RCDs). These are protection elements often used in electrical industrial installations. The paper intends to explain, in a very simple manner, the following aspects related to the residual current devices:

- Constituting elements and operation principles.

- Basic specifications.

- Characteristic curves.

- Types of RCDs.

- Applications.

This work is devoted to students or professionals that are involved in electrical installations design and, more specifically, in the basic switchgear used in those installations.

\section{Introduction}

Today, RCDs are recognized as the most effective means of protecting life and property against electrical hazards in low voltage systems. RCDs monitor the residual current in the protected circuit (the residual current is the sum of the instantaneous values of all currents flowing through all active conductors in a circuit at a defined point in the electrical system). An RCD will trip when the residual current is detected in excess of the trip rating of the device.

The idea is that these devices should break the circuit when the residual current reaches a value that can be potentially dangerous for the users and not for the 'normal' currents that can flow to ground or external conductive parts (leakage currents) that are usually much lower.

Specific information on L.V. Fuses can be found in the norm UNE 20-383-75.

\section{Objetives}

The following learning objectives are pursued in this paper:

- To understand the operation of the basic components of a RCD.

- To know the basic terms defining a RCD and enabling its specification.

- To understand the characteristic curves of a RCD.

- To be able to describe and compare the different types of RCDs. 


\section{Development}

\subsection{Basic parts and operation principle}

The basic element of a RCD is a current transformer based on a toroid that surrounds the live conductors (phases and neutral) and detects the algebraic sum of the current in these live conductors. The toroid winding detects variations in the flux induced by the residual current.

During normal operation (no insulation fault), the algebraic sum of the current in the live conductors is equal to zero and the toroid does not detect any net flux. If an insulation fault occurs in the system to be protected, the sum is no longer equal to zero and the fault current in the toroid generates a current in the winding.

This current is rectified, filtered and amplified. If the resulting signal is greater than a set threshold, a time delay is initiated (it may be equal to zero for an instantaneous response). If the fault is still present at the end of the time delay, it is provoked the disconnection of the circuit by means of the holding magnet tripping unit and the latching mechanism (Figure 1) [4, 5].

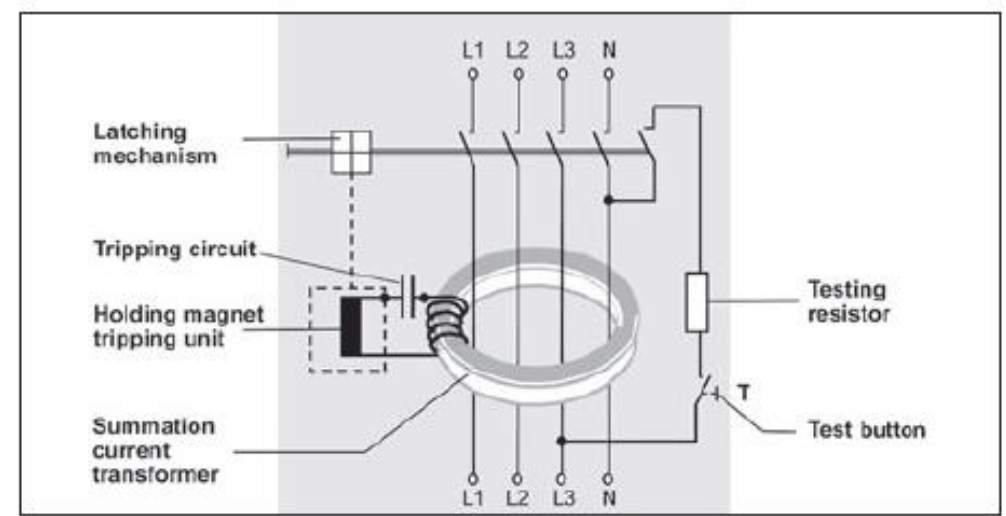

Figure 1. Main elements of a RCD (Source: [5])

The functionality of the RCD can be tested using the test button available on every device. Pressing the test button generates an artificial residual current which must trip the RCD. In order to guarantee protection against dangerous shock currents, the reliability of the RCD must be tested when the installation is put into operation and at regular intervals [5].

\subsection{Basic specifications}

Some basic characteristics defining a Residual Current Device are explained next [4]:

- Sensitivity $\left(I_{\Delta n}\right): R C D$ sensitivity is expressed as the rated residual operating current, noted $I_{\Delta n}$. It is defined as the residual current that guarantees the tripping of the RCD. Preferred values have been defined by the IEC, thus making it possible to divide RCDs into three groups according to their $I_{\Delta n}$ value:

- High sensitivity (HS): 6-10-30 mA, 
- Medium sensitivity (MS): 0.1-0.3-0.5-1 A,

- Low sensitivity (LS): 3-10-30 A.

RCDs for residential or similar applications are usually high sensitivity. In industrial applications and other special uses are typical the ones with medium and Low sensitivities.

- Rated non-operating residual current (Inf): it is the value of residual current below which it is guaranteed that the RCD will never trip. It is usually equal to $0{ }^{\prime} 5 \cdot I_{\Delta n}$.

- Break time: According to the break time, there are two groups of devices [4]:

- $G$ (general use) for instantaneous RCDs (i.e. without a time delay)

- $S$ (selective) or T (time delayed) for RCDs with a short time delay (typically used in circuits containing surge suppressors)

- $\quad$ Surge current resistance: The surge current refers to the peak current an RCD is designed to withstand using a test impulse of specified characteristics (an 8/20 $\mu \mathrm{s}$ impulse, named after the time constants of the rise and fall of current).

\subsection{Characteristic curves}

\subsubsection{Time-current characteristic curves}

These curves provide the tripping time of the RCD versus the residual current in the protected part of the installation [1]. Figure 2 shows these curves for RCDs with sensitivities $\left(I_{\Delta n}\right) 10,30$ and $300 \mathrm{~mA}$. It is shown how, if the residual current is lower than the rated residual non-operating current $\left(\ln _{n}=1 / 2 \cdot I_{\Delta n}\right)$, the RCD will never trip. If the residual current is between $I_{n f}$ and $I_{\Delta n}$, it can trip or not, but it is sure that it will trip for currents higher or equal to $I_{\Delta n}$. Typically, the tripping time is very short $(<30 \mathrm{~ms})$, unless the RCD is selective (delayed).

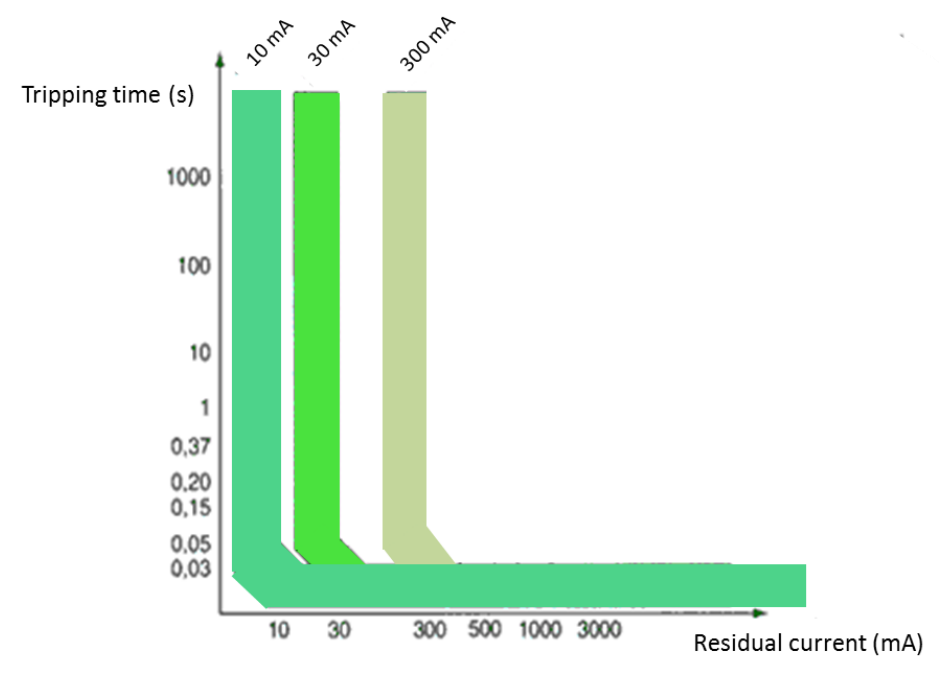

Figure 2. Time-current characteristic curve of a RCD 


\subsubsection{RCDs selectivity}

In order to reach selectivity between two RCD in series, the $1 / t$ characteristic curve of the RCD upstream (DI in Figure 3) must be above and on the right of the RCD downstream (D2 in Figure 3) [1]. This condition is graphically depicted in Figure 4.

This graphical condition is achieved always that the following two requirements are fulfilled:

- The sensitivity of D2 $\left(I_{\Delta^{n}, D 2}\right)$ is lower than the rated residual non-operating current of DI $(\ln , D 1)$ :

$$
\mathrm{I}_{\Delta \mathrm{n}, \mathrm{D} 2}<\mathrm{I}_{\mathrm{nf}, \mathrm{D} 1}=\frac{1}{2} \cdot \mathrm{I}_{\Delta \mathrm{n}, \mathrm{D} 1}
$$

- D1 is selective (i.e. delayed); this means that, when a residual current appears, Dl waits a certain time $t_{r}$ before starting the tripping process.

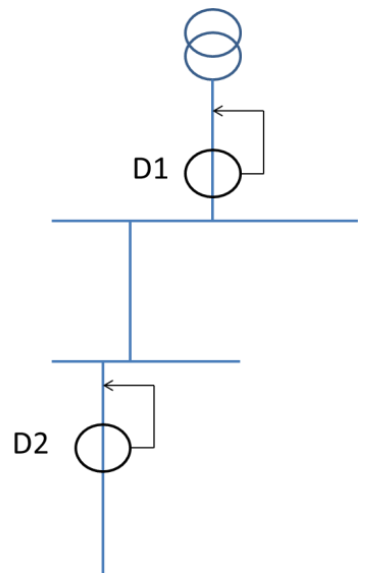

Figure 3. Situation of D1 and D2

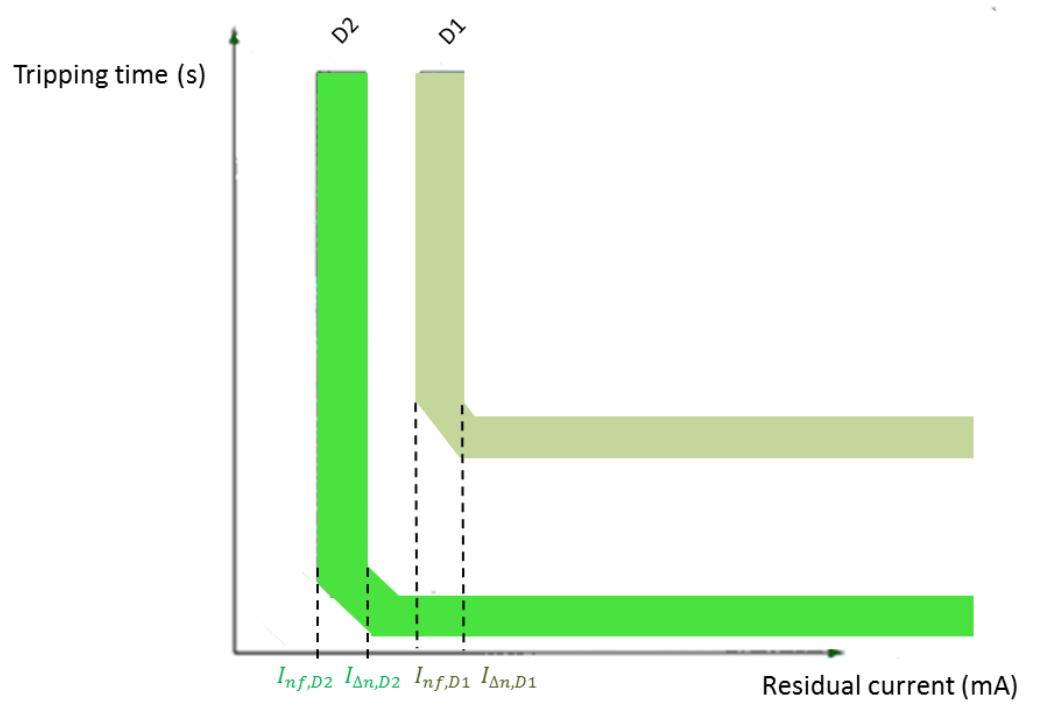

Figure 4. Graphical representation for the selectivity condition between D1 and D2 


\subsection{Types of RCDs}

Standard IEC 60755 (General requirements for residual current operated protective devices) defines three main types of RCD depending on the characteristics of the fault current.

- Type AC: RCD for which tripping is ensured for residual sinusoidal alternating currents.

- Type A: RCD for which tripping is ensured for residual sinusoidal alternating currents, for residual pulsating direct currents, for residual pulsating direct currents superimposed by a smooth direct current of $0.006 \mathrm{~A}$, with or without phase-angle control, independent of the polarity

- Type B: RCD for which tripping is ensured as for type A, for residual sinusoidal currents up to $1000 \mathrm{~Hz}$, for residual sinusoidal currents superposed by a pure direct current, for pulsating direct currents superposed by a pure direct current, for residual currents which may result from rectifying circuits (three pulse star connection or six pulse bridge connection, two pulse bridge connection line-toline with or without phase-angle monitoring, independently of the polarity.

\subsection{Applications}

Among the most important applications of the RCDs, we can remark:

- Protection against indirect contacts: Indirect contact is the contact of a human body with a not live but electrically conductive part. In these cases, the demand is for automatic disconnection of the power supply when a fault can pose a risk due to the intensity and duration of the touch voltage. Residual current protective devices with rated residual currents of over $30 \mathrm{~mA}$ are also suitable for this purpose. In order to achieve the protective effect, the tripping conditions must be complied with. In addition, the dangerous touch voltage must not be present for an impermissible length of time, whereby account must be taken of the grounding resistance and the rated residual current [5].

- Complementary protection against direct contacts (high-sensitivity RCD): Direct contact is understood to mean direct contact of a human body with a live part. In the case of a direct contact, a current flows though the human body closing the circuit through the transformer neutral earthing. This 'residual current' in the circuit can be detected by a RCD breaking the circuit and, hence, removing the direct contact. If the RCD has high-sensitivity, in a typical low voltage installation (phase voltage $230 \mathrm{~V}$ ), it will provide a line disconnection such that the body exposure to the current will be not dangerous [1].

In spite of this fact, since it does not prevent the circulation of a certain current through the body, this should not be the only way of providing protection against electric shock and does not replace implementation of a further protection measure as required by IEC 60364-4-41 or HD 60364-4-41 [5].

- Fire protection [5]: for "locations exposed to fire hazards", IEC 60364-4-482 or HD 60364.4.482 requires measures for the prevention of fires which can be caused by insulation faults. According to this standard, cables and lines in TN and TT systems must be protected by residual current protective devices with a rated 
residual current of $\mathrm{I}_{\Delta \mathrm{n}} \leq 300 \mathrm{~mA}$. Exceptions are mineralized lines and busbar systems. In applications in which resistive faults can cause a fire (e.g. radiant ceiling heating with panel heating elements), the rated residual current must be $\mathrm{I}_{\Delta \mathrm{n}}=30 \mathrm{~mA}$. The fire protection provided by residual current protective devices, however, should not be limited to locations exposed to fire hazards but should always be provided.

\section{Conclusions}

This work constitutes a brief introduction to the RCDs. The work explains the basic elements of a RCD and its operation principles. Moreover, the basic quantities used for the specification of a RCD are enumerated. The work describes the main characteristic curves of a RCD as well as the necessary conditions to achieve selectivity between RCDs. The RCD typology is also analyzed in the paper. Finally, the main applications of the RCDs are described.

\section{References}

\subsection{Textbooks:}

[1] Roger, J.; Riera, M.; Roldán, C.: "Tecnología Eléctrica", Ed. Síntesis, 2010, Cap. 8.

[2] Roger, J.; Riera, M; Roldán, C.; Antonino, J.A.: "Exercises and questions". Editorial UPV. 2010.

[3] Reglamento Electrotécnico de B.T. (R.B.T.2002) (MIE-BT).

\subsection{Technical catalogs:}

[4] Merlin Gerin, Cahier technique no. 114, "Residual current devices in LV", Jaques Schonek. February 2006.

[5] Siemens AG, Industry Sector, "Residual Current Protective Devices: BETA LowVoltage Circuit Protection Technology Primer". Germany, 2010.

[6] ABB, technical data sheet - Miniature circuit breakers \& Residual-currentoperated circuit-breakers. 